Hautarzt 2020 $\cdot 71: 657-659$

https://doi.org/10.1007/s00105-020-04665-5

(C) Springer Medizin Verlag GmbH, ein Teil von Springer Nature 2020

\section{R. Kaufmann' ${ }^{1}$ A. Navarini ${ }^{2}$}

'Klinik für Dermatologie, Venerologie und Allergologie, Universitätsklinikum Frankfurt, Frankfurt am Main, Deutschland

${ }^{2}$ Klinik für Dermatologie, Universitätsspital Basel, Basel, Schweiz

\title{
Künstliche Intelligenz - Es kommt darauf an, was wir daraus machen
}

Liebe Leserinnen und Leser,

wie alle Menschen sind auch wir als Dermatologinnen und Dermatologen begrenzt belastbar, dürfen ermüden und dürfen irren. Wir lernen, verstehen, erinnern und vergessen.

Dennoch: Individuell ausgeprägte natürliche Intelligenz und Begabungen, Einfallsreichtum und gestalterische Initiative haben der Menschheit nicht nur Werkzeuge und Maschinen beschert, sondern ungeahnte Möglichkeiten einer hoch entwickelten Technisierung, mit der wir die uns gesetzten Grenzen zerebraler Befähigung und körperlicher Leistung überwinden. Seit mehreren Jahren bereits befinden wir uns darüber hinaus in einer Phase der digitalen Transformation, in der nun zunehmend künstliche Intelligenz (KI) in ganz unterschiedlichen Facetten global und zeitlich unbegrenzt Einfluss auf unsere Entscheidungen nimmt und in der Bewältigung des privaten wie beruflichen Alltags nicht mehr wegzudenken ist. Beflügelt wird diese rasante Entwicklung aktuell infolge der Pandemie, wo Videokonferenzen oder telemedizinische Sprechstunden, digitale Lehre und vieles mehr unsere Gegenwart nicht nur verändern, sondern den Ablauf solcher Zusammenkünfte und Veranstaltungen erst ermöglichen. Gleichzeitig drohen Szenarien des Kontrollverlustes und Missbrauchs, so beispielsweise bei der diesbezüglich in den öffentlichen Fokus geratenen Gesichtserkennungssoftware. Digitalisierung und künstliche Intelligenz haben vor der Dermatologie nicht
Halt gemacht und werden absehbar unser Berufsbild und unsere Arbeitsabläufe mit Sicherheit verändern. Die Weichen jedenfalls sind gestellt.

\section{》) Digitalisierung und künstliche Intelligenz werden unser Berufsbild und unsere Arbeitsabläufe verändern}

„Dermatologie wird Morphologie sein, oder sie wird nicht sein“, so seinerzeit das dermatologische Weltbild von Oscar Gans. In diesem Kontext zitierte er gerne Goethes Worte, wonach es für uns Menschen ja gerade das Schwerste von allem sei, was einem das Leichteste dünke, nämlich mit den Augen zu sehen, was vor unseren Augen liegt. So ist es nicht verwunderlich, dass gerade unser visuell morphologisch geprägtes Fach - genauso wie dasjenige der Pathologie oder der Radiologie - von den Möglichkeiten einer sinnvoll genutzten computerassistierten Diagnostik oder einer telemedizinischen Versorgung in besonderem Maße profitieren kann. Enorme Rechnergeschwindigkeiten, schier endlose Datenspeicherkapazitäten zusammen mit einer immer höher auflösenden Bilderdokumentation haben der künstlichen Intelligenz mit ihren ausgeklügelten Algorithmen zur computerbasierten Bildanalyse den Weg bereitet.

Daher gilt es auch gerade für uns, potenziellen Nutzen, mögliche Nachteile für das Fachgebiet, juristische Aspekte der Datensicher- und -hoheit und letztlich 
die Tauglichkeit möglicher Einsatzszenarien für den Klinik- und Praxisalltag zu eruieren. Wie bei vielen technologischen Innovationen kommt es letztlich darauf an, was wir daraus machen. Oberste Maxime sollte dabei sein, sich von natürlicher Intelligenz leiten zu lassen und sich die Möglichkeiten der künstlichen Intelligenz zum Vorteil für Patienten und das Fachgebiet nutzbar zu machen, um letztlich einen tatsächlichen Mehrwert für alle Beteiligten $\mathrm{zu}$ generieren.

So ist es uns als Herausgebern dieses Leitthemenheftes ein besonderes Anliegen, den aktuellen Stellenwert der künstlichen Intelligenz aus verschiedenen Blickwinkeln der Dermatologie zu beleuchten. Es freut uns daher sehr, dass wir hierzu namhafte Autoren und Kenner der Materie für entsprechende Beiträge gewinnen konnten, die sich in ihren Arbeiten thematisch mit den theoretischen Grundlagen, aber auch den unterschiedlichen praxisrelevanten Aspekten dieses faszinierenden Gebietes befassen. Wir wünschen Ihnen eine interessante und erkenntnisreiche Lektüre der vorliegenden Ausgabe.

Ihre

Roland Kaufmann

Alexander Navarini

\section{Korrespondenzadresse}

Prof. Dr. R. Kaufmann
Klinik für Dermatologie,
Venerologie und Allergologie,
Universitätsklinikum
Frankfurt
Theodor-Stern-Kai 7,
60590 Frankfurt am Main,
Deutschland
Roland.Kaufmann@kgu.de

Prof. Dr. A. Navarini
Klinik für Dermatologie,
Universitätsspital Basel
Spitalstr. 21/Petersgraben 4,
4031 Basel, Schweiz
alexander.navarini@usb.ch

Interessenkonflikt. R. Kaufmann und A. Navarini geben an, dass kein Interessenkonflikt besteht.

\section{H.-G. Nobis, R. Rolke, T. Graf-Baumann}

\section{Schmerz - eine Herausforderung}

Ein Ratgeber für Betroffene und Angehörige

Springer Medizin 2020, 3., vollständig aktualisierte und erweiterte Auflage, 204 S., 52 Abb., (ISBN: 978-3-662-60400-7), Softcover 19,99 EUR

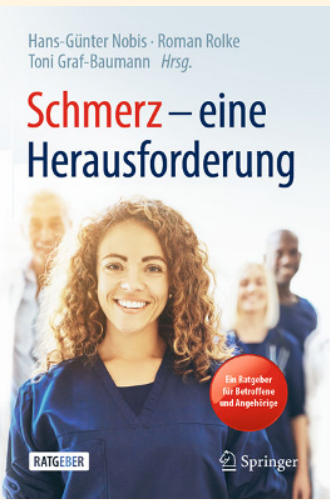

Mit einem freundlichen Lächeln präsentiert sich die 3. vollständig aktualisierte und erweiterte Auflage des bewährten Ratgebers für Betroffene und Angehörige.

Den Herausgebern Herrn Dipl. Psych. HansGünter Nobis, Herrn Univ.-Prof. Dr. med. Roman Rolke und Herrn Prof. Dr. med. Toni GrafBaumann ist es gelungen, über 50 namhafte Schmerzexperten für Beiträge zu 70 SchmerzThemen zu gewinnen. Diese Mühe hat sich gelohnt. Die aktuelle Auflage zeichnet sich durch gelungene Erweiterungen aus, indem einige Kapitel neu eingefügt sind und $\mathrm{Ab}$ schnitte nach neuesten wissenschaftlichen Erkenntnissen überarbeitet wurden.

Das Buch richtet zunächst den Fokus auf die Herausforderung für das Verständnis und die verschiedenen Ursachen von Schmerzen. Es wird klar und verständlich vermittelt, dass die Betroffenen einen wesentlichen Beitrag zu einer erfolgreichen Behandlung beitragen können und gemeinsam mit Behandlern das Therapieziel verfolgen. Bereits auf den ersten Seiten wird die Motivation der Betroffenen hierfür gefördert, sich gemeinsam mit Ärzten und Therapeuten aus den Disziplinen Psychologie und Physiotherapie aktiv an den Therapien zu beteiligen. Die beleuchteten Hintergründe, um ein Verständnis für die Schmerzerkrankung zu erreichen, sind übersichtlich, klar, einfühlsam und verständlich geschrieben.

Im speziellen Teil werden die wesentlichen und häufigsten chronischen Schmerzerkrankungen in didaktisch guter Weise beschrieben und bieten eine rasche Möglichkeit sich zu informieren. Die Bereiche "Schmerz und Sexualität", "Wachstumsschmerzen bei Kindern“, „Endometriose“ und "Gelenkschmerz", ebenso wie "Naturheilkunde bei Schmerz" und medikamentöse Besonderheiten bei Schwangerschaft, Stillzeit auf Reisen und beim Sport sind umfassend erweitert und aktualisiert.

Neben den Besonderheiten bei Schmerz, der Diagnostik und den therapeutischen Möglichkeiten ist das Kapitel „Erfahrungsberichte von Schmerzpatienten" für Betroffene und Angehörige sehr bereichernd. Schmerzpatienten unterschiedlichen Alters und mit verschiedenen chronischen Schmerzerkrankungen berichten über ihre persönliche Leidensgeschichte. Diese hautnahen Berichte sind lebensnah und eingängig, sie machen Mut auch in scheinbarer Hoffnungslosigkeit mit einer Schmerzerkrankung zu leben und eröffnen Perspektiven im Umgang damit. Gleichzeitig vermitteln diese Berichte auch uns Behandlern, wie wichtig unsere empathische Professionalität für diese Patienten ist. Es macht auch uns Behandlern Mut, auch wenn wir dies im arbeitsreichen Alltag und möglichen Auseinandersetzungen mit übergeordneten Strukturen nicht täglich wahrnehmen.

Die beiden letzten Kapitel beschreiben übersichtlich welche Versorgungsstrukturen für Patienten zur Verfügung stehen und verfolgt werden können, der anschließende Serviceteil lässt keine Wünsche offen hinsichtlich Informationen inklusive eines Glossars zum Nachschlagen medizinischer Fachbegriffe. Das Buch ist eine lesenswerte Quelle an Hilfen, Erklärungen und Anregungen zur Schmerztherapie allgemein und zu verschiedenen Schmerzerkrankungen. Auch wenn der rote Button auf dem Cover sich an Betroffene und Angehörige richtet, so bietet dieses Buch auch dem medizinisch interessierten Laien, wie auch den Profis im Alltag patientengerechte Erklärungen zu wesentlichen Aspekten von Schmerzerkrankungen.

PD Dr. med. Rita Laufenberg-Feldmann, Mainz 
Hier steht eine Anzeige.

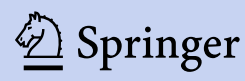

